\title{
Research and improvement of GPON dynamic bandwidth allocation algorithm
}

\author{
Qiang Han ${ }^{\mathrm{a}}$, Hanjian $\mathrm{Ni}^{\mathrm{b}}$ \\ China Satellite Maritime Tracking and Control Department, Jiangyin 214431, China \\ a1510603630@qq.com, b2794049921@qq.com
}

Keywords:GPON, DBA, utilization.

Abstract. The dynamic bandwidth allocation algorithm for GPON system based on traffic detection is proposed, and the algorithm of dynamic allocation is studied. According to the existing problems, the improved scheme is put forward.

\section{Introduction}

GPON is a high bandwidth, full service system, a PON port can be hung up 128 sets of ONT equipment. However, the downlink of GPON system is broadcast, and the uplink is in accordance with the way of time division multiplexing to allocate bandwidth to each ONT device, and the total bandwidth of each device is limited by technology and utilization, the system will not allow all ONT devices to achieve the highest bandwidth, so the allocation mechanism of uplink bandwidth is crucial.

\section{Bandwidth allocation algorithm analysis}

\subsection{Time division multiplexing of uplink channels.}

In GPON networks, multiple ONU is connected to a PON port of OLT, in order to make the ONU data do not conflict, the uplink uses time division multiplexing. Each ONU is obtained from the channel and the duration of the OLT is responsible for scheduling, for this OLT need to implement the dynamic bandwidth allocation algorithm (DBA), the calculation of the distribution bandwidth of each ONU, and then the corresponding channel access rules to notify ONU by way of time slot. After the ONU is obtained, the local data is transmitted in the form of the corresponding authorization time slot. An excellent DBA algorithm is required to track the changes in data traffic, as required. If the distribution value is too large, it will cause bandwidth waste, so that the channel utilization rate is reduced; if the allocation value is too small, it will make business delay increases. The implementation of the DBA algorithm is in charge of OLT, and the input of the algorithm is the amount of data in the T-CONT queue. According to the demand of the buffer capacity, the bandwidth of OLT is calculated by T-CONT, and finally, the bandwidth allocation rules of ONU are calculated by DBA in the form of BWmap.

\subsection{OLT scheduling.}

The object of the DBA algorithm is not ONU, but ONU (T-CONT). An ONU can have more than one T-CONT, each T-CONT is granted a full net only Alloc-ID. Each ONU has a fixed number of T-CONT and each T-CONT carries a set of logical connections. In ONU on-line registration, the OLT using omcc ascertain the number of ONU t-cont, and via ploam message for the t-cont distribution only Alloc-ID. Alloc-ID is 12bit, each ONU has at least one default Alloc-ID, the value is equal to its ONU-ID, and the default Alloc-ID can load PLOAM and OMCC data. OLT sends a channel access to the ONU, the rule is achieved by a specific field of the downlink GTC frame header, and this specific field is called BWmap. BWmap within a number of authorized time slots, each authorized time slot corresponding to a T-CONT, the T-CONT Alloc-ID Start and the Time Stop (Time) and the end of the channel are specified in the authorization time slot. The unit is byte at the moment, at the beginning of an uplink frame, as a result of an uplink frame of 125us, 19440 bytes, so the accuracy of the DBA allocation bandwidth is 64kbit/s. After receiving the OLT BWmap ONU, the is resolved to determine the size of the authorization obtained by the T-CONT, and to transmit the 
burst frame at the start time of the uplink frame. Each T-CONT in the corresponding time slot in the frame to send, until the end of the arrival of the moment.

\subsection{ONU Report.}

DBA algorithm is based on the bandwidth allocation of the ONU within the T-CONT of the number of bytes, the number of cache, it means that before the bandwidth is insufficient, need to allocate more bandwidth value. ONU in the DBRu BURST field to the OLT T-CONT of the BWmap, OLT can be used to control the FLAG ONU. ONU statistics T-CONT buffer accounted for the amount, the above reported block for the OLT to report the cache capacity. On a PON port, the reported block size must be consistent, the size of the ONU registration on the line, passing through the OMCI channel, the default size of 48 bytes. Cache footprint calculation process is: first accumulated t-cont packet size, total number of bytes, divided by the block size and rounding up. Like type (1).

$$
R=\operatorname{ceil}\left(\frac{1}{E} \sum_{i=1}^{k} L_{i}\right)
$$

$\mathrm{K}$ is the number of data packets; the size of each packet is represented by the size of the unit byte; $B$ is the size of the $R$.

\section{Bandwidth allocation model}

In order to describe the traffic flow of T-CONT, defining the demand rate, representing a fixed time interval, the T-CONT in order to empty the required average rate, unit bit/s. Like type (2). At the time of the T cache, the amount of data that is new to the T-CONT in the time interval is bit.

$$
R_{\mathrm{L}}(\mathrm{t})=\frac{B(\mathrm{t})+\mathrm{A}(\mathrm{t}, \mathrm{t}+\Delta)}{\Delta}(2)
$$

Each T-CONT is given a business descriptor, which specifies four bandwidth parameters: fixed bandwidth, bandwidth, maximum bandwidth, and additional bandwidth. I for T-CONT, Alloc-ID, the business descriptors for $D^{i}=\left(R_{F}^{i}, R_{A}^{i}, R_{M}^{i}, \chi_{A B}^{i}\right)$

$\mathrm{R}_{\mathrm{F}}$ fixed bandwidth, unit bit/s, which represents the reserved portion of the uplink bandwidth, does not carry out dynamic bandwidth allocation. Regardless of traffic flow size, regardless of the load size of the PON port, the OLT allocates constant bandwidth values for the fixed bandwidth T-CONT. $\mathrm{R}_{\mathrm{A}}$ ensure bandwidth, the unit bit/s, as long as the T-CONT business is not enough bandwidth, regardless of the PON port load size, OLT are allocated to their demand value. $\mathrm{R}_{\mathrm{M}}$ maximum bandwidth, unit bit/s, represents the maximum bandwidth that a T-CONT can get in any case. Indicates an additional bandwidth, with three state properties: \{None, NAB, BE\},Respectively, $\chi_{A B}$ additional bandwidth, additional bandwidth for the type of non guaranteed type or additional bandwidth for the best type. A correct business descriptor, whose parameters are required to meet (3).

$R_{F}^{i}+R_{A}^{i} \leq R_{M}^{i}(3)$

\section{Existing algorithms introduced}

\subsection{Algorithm content.}

DBA algorithm based on T-CONT hierarchical polling, the total bandwidth of the uplink channel is represented by the maximum byte number 19440, which is FB, and the total bandwidth of the burst header is deducted, the overhead of the $\mathrm{DBRu}$, the space overhead, the PLOAM overhead, and the. OLT gets the T-CONT from the DBRu field of each burst frame, and will be reported as a bandwidth requirement. Next, the T-CONT1 is allocated to the fixed bandwidth of T-CONT1, T-CONT4, T-CONT2, T-CONT3, and T-CONT2, and the bandwidth is allocated to T-CONT3, which is used to ensure the bandwidth allocation, and the T-CONT4, which has the lowest priority, is allocated to the lowest priority.

For T-CONT's demand for bandwidth improvement, the demand bandwidth is not directly used DBRu reported value, but the traffic forecast. Because of the T-CONT reporting requirements and the 
presence of OLT in the presence of bandwidth and transmission delay, bandwidth scheduling cannot be carried out in real time, so that the error between the distribution and demand. DBA predicts that in the report and the following period of time to reach the T-CONT of new traffic, the new traffic plus the reported value is the demand bandwidth. The traffic prediction uses the linear prediction model, because the network traffic has self-similar characteristics, so the use of historical traffic value to predict future traffic, in order to obtain a relatively accurate T-CONT bandwidth demand.

\subsection{Algorithm in the realization of the problem.}

The problem of the complexity of the algorithm is not considered. A frame of time length of 125us, rely on the processor is difficult to perform the allocation algorithm, the general OLT device at least need to support hundreds of T-CONT, computing time is very tight.

Existing DBA studies have not considered the impact of FEC encoding on the bandwidth allocation, in the simulation model, all FEC ONU attributes are closed state. If you open the ONU FEC attributes, will bring great impact on the existing algorithms: as a result of the additional code block, the quantitative data, and ONU reported value of t-cont cache bytes is DBRu, not encoded, is also an effective load, if in accordance with the reported value to allocate the bandwidth, bandwidth obviously partial small.

In addition to the effective bandwidth of the uplink channel, there are a lot of protocol overhead, including: FEC encoding overhead, the head overhead, the $C$ overhead, the overhead of the burst, the overhead of the $\mathrm{DBRu}$, the overhead of the overhead. But in fact, the cost of the value will be changed with the distribution, if the direct deduction of a maximum fixed value, it is clear that a waste of bandwidth, so it is necessary to make full use of this part of the bandwidth.

In the G.984.3 standard, ONU is required to open the window to complete the sequence number and range. During this period, the DBA cannot allocate the bandwidth, the uplink channel is idle, so the traffic delay will increase, and the algorithm need to consider the impact of the window to the algorithm.

DBA is calculated from the distribution of bandwidth value cannot be directly to the ONU broadcast, need to be transformed into the BWmap Start Stop (Time) and the end of the time (Time), so we need to design additional algorithm to complete this part of the work.

\section{Improved algorithm}

Because of the complexity of the algorithm, the T-CONT needs to get a bandwidth and delay will be increased by a number of upstream frames, so it is proposed to solve the problem of single multi frame T-CONT. The time of each calculation and the allocation of a bandwidth is called a computation period, which can be configured for 2-32 uplink frame (125us). Let DBA calculate the cycle to experience the $\mathrm{N}$ uplink frame, the bandwidth allocated for T-CONT can appear in each of the uplink frame, but also in the $\mathrm{N}$ uplink frame only appear once, which will be divided into two categories: single frame and multi frame T-CONT. For single frame T-CONT, each of the uplink frame for its allocation of bandwidth, so the number of transmission, the interval is small, the delay is smaller, but due to each transmit once, with a frame head and the space overhead, so reduce the efficiency; for multi frame T-CONT, it is just the opposite, the number of transmission is small, so the delay is large, but increased efficiency, reduce bandwidth waste. DBA provides an optional single frame and multi frame mode for the user to select the delay and utilization as an option.

On the basis of the computing period, the concept of B is also presented, which is a compromise between single frame and multi frame. Single frame each frame is sent, multiple frames a calculation cycle to send a. With the next cycle, it can make the transmission frequency between the two, improve the flexibility. For a single frame T-CONT, the time interval of the adjacent two authorization will be smaller, and the delay time is reduced.

Algorithm according to the protocol requirements, the ONU on the line registration required to open the window, not assigned any authorization, in order to guarantee the high quality of service delay, the loss of bandwidth to compensate for the window period. So the T-CONT type, fixed position type and T-CONT type TDM, are defined, and the bandwidth is compensated by the OLT open window, which gives the equivalent loss bandwidth. 


\section{Test analysis}

An OLT splitter with multiple ONU connections, all ONU Ethernet interface and network analyzer, OLT Ethernet interface with the same network analyzer, thereby forming a closed loop. The network analyzer is responsible for sending the Ethernet packets to ONU, and the OLT, and then return to the analyzer.

In the GPON uplink, the background traffic is injected into each ONU, so that the uplink is close to full load. After that, to an ONU injection of additional traffic, so that the channel is full load. Full flag is no packet loss, and the delay has not significantly changed, at this time to get the maximum throughput of PON. ONU total of 33, all through the optical fiber connected to an OLT. The 33 ONU all configured a default T-CONT: bandwidth type for FIX, traffic 0M, and configuration bandwidth 1M. The first 32 ONU each configuration of a TYPE3 type T-CONT:AB configuration bandwidth $1 \mathrm{M}$, the maximum bandwidth of $1 \mathrm{G}$, the flow is constant for $25 \mathrm{M}$. At this time, the total flow rate is $800 \mathrm{M}$, and then to the thirty-third ONU injection of additional traffic, in different packet length and single multi frame configuration, so that the uplink channel is loaded, the throughput and delay data. The calculation cycle and the next cycle are 8 frames, which are divided into two groups. The first group T-CONT is configured as a single frame, which is configured as a single frame and a multi frame conversion, respectively, to test the full load throughput and packet delay in different cases, such as table 1 . The packet length is 512 bytes, 1024 bytes, 1280 bytes, 1518 bytes, and the mixture of the length of the packet.

Table 1

\begin{tabular}{ccccccc}
\hline $\begin{array}{c}\text { Computation } \\
\text { cycle }\end{array}$ & $\begin{array}{c}\text { single multi } \\
\text { frame } \\
\text { adaptive }\end{array}$ & $\begin{array}{c}\text { distribution } \\
\text { cycle }\end{array}$ & $\begin{array}{c}\text { test packet } \\
\text { length(byte) }\end{array}$ & $\begin{array}{c}\text { additional } \\
\text { traffic(Mb/s) }\end{array}$ & $\begin{array}{c}\text { total } \\
\text { upstream } \\
\text { traffic(Mb/s) }\end{array}$ & delay(us) \\
\hline \multirow{3}{*}{8 frame } & & & 512 & 352 & 1152 & 256.558 \\
& adaptive & 8 frame & 1024 & 335 & 1135 & 258.652 \\
& & & 1518 & 331 & 1131 & 306.325 \\
& & & Mixed & 331 & 1131 & 306.325 \\
\hline
\end{tabular}

Table 2

\begin{tabular}{|c|c|c|c|c|c|c|}
\hline $\begin{array}{l}\text { Computation } \\
\text { cycle }\end{array}$ & $\begin{array}{l}\text { single multi } \\
\text { frame } \\
\text { adaptive }\end{array}$ & $\begin{array}{l}\text { distribution } \\
\text { cycle }\end{array}$ & $\begin{array}{l}\text { test packet } \\
\text { length(byte) }\end{array}$ & $\begin{array}{c}\text { additional } \\
\text { traffic(Mb/s) }\end{array}$ & $\begin{array}{c}\text { total } \\
\text { upstream } \\
\text { traffic(Mb/s) }\end{array}$ & delay(us) \\
\hline \multirow{5}{*}{8 frame } & \multirow{5}{*}{ multi frame } & \multirow{5}{*}{8 frame } & 512 & 425 & 1225 & 704.391 \\
\hline & & & 1024 & 410 & 1210 & 752.338 \\
\hline & & & 1280 & 395 & 1195 & 579.572 \\
\hline & & & 1518 & 400 & 1200 & 521.501 \\
\hline & & & Mixed & 456 & 1256 & 779.911 \\
\hline
\end{tabular}

For second sets of tests, the change of single multi frame configuration, all T-CONT configuration for multi frame, other parameters are unchanged, the test data is shown in table 2. Compared with the first and two groups of data, it can be seen that the first group is configured as a single frame, the smallest delay mode, the throughput is small, the delay is small; the second group is configured for multi frame, which improves the throughput and the delay time.

\section{Summary}

First of all, the paper introduces the DBA algorithm, introduces the GPON uplink channel multiplexing rule, and then describes the existing DBA research results, analyzes the existing problems, and puts forward the improvement strategy, introduces the improved algorithm. Finally, the performance of the DBA algorithm in the actual scene is tested. 


\section{References}

[1]. F. Selmanovic, E. Skaljo. GPON in Telecommunication Network [C]. Ultra ModernTelecommunications and Control Systems and Workshops, Congress, 2010, 1012-1016

[2]. Qiang, Zhou virtual optical access network technology and its application [M] Beijing: Tsinghua University Press, 2005, 119-144

[3]. F. Effenberger, TS El-Bawab Passive optical networks (PONs):Past, present, and future [J] .Optical Switching and Networking, 2009, 6 (3): 143-150

[4]. Pingchen Wei, Tang Yuliang on next generation passive optical network [J] Xiamen Institute of Technology, 2005, 12, Vol3:. 48-50

[5]. ML Bonilla, FR Barbosa, E. Moschim Techno-economical comparison between GPON and EPON networks [C] International Telecommunication Union - Proceedings of the 2009 ITU-T Kaleidoscope Academic Conference, 2009, 201-205

[6]. I. Cale, A. Salihovic, M. Ivekovic. Gigabit passive optical network-GPON [C]. Information Technology Interfaces, 2007. ITI 2007. 29th International Conference on. IEEE, 2007,679-684

[7]. ITU-T Recommendation G.984.2 Gigabit-capable passive optical networks (GPON): Physical media dependent (PMD) layer specification [S] Geneva:2008

[8]. F. Effenberge, D. Clearly, O. Haran, et al An introduction to PON technologies [J] .Communications Magazine, IEEE, 2007, 45 (3): S17-S25

[9]. Y. Guofeng Discussion on the Application of GPON Technology in Access Network [J] .Electric Railway, 2009, 1:. 012

[10]. A. Cauvin, A. Tofanelli, J. Lorentzen, et al Common technical specification of the G-PON system among major worldwide access carriers [J] Communications Magazine, IEEE, 2006,44 (10): 34- 40 